\title{
Removing the Twin Image in Digital Holography by Segmented Filtering of In-focus Twin Image
}

\author{
C. McElhinney ${ }^{a}$, B. M. Hennelly ${ }^{a}$, L. Ahrenberg ${ }^{a}$ and T. J. Naughton ${ }^{a, b}$ \\ ${ }^{a}$ Department of Computer Science, National University of Ireland, \\ Maynooth, Ireland; \\ ${ }^{b}$ University of Oulu, RFMedia Laboratory, Oulu Southern Institute, \\ Vierimaantie 5, 84100 Ylivieska, Finland
}

\begin{abstract}
We propose and investigate a new digital method for the reduction of twin-image noise from digital Fresnel holograms. For the case of in-line Fresnel holography the unwanted twin is present as a highly corruptive noise when the object image is numerically reconstructed. We propose to firstly reconstruct the unwanted twin-image when it is in-focus and in this plane we calculate a segmentation mask that borders this in focus image. The twin-image is then segmented and removed by simple spatial filtering. The resulting digital wavefield is the inverse propagated to the desired object image plane. The image is free of the twin-image resulting in improved quality reconstructions. We demonstrate the segmentation and removal of the unwanted twin-image from in-line digital holograms containing real-world macroscopic objects. We offer suggestions for its rapid computational implementation.
\end{abstract}

Keywords: Twin-image reduction, Digital Holography, Segmentation

\section{INTRODUCTION}

Since the invention of holography by Gabor ${ }^{1}$ in 1948, the removal of the unwanted twin-image has remained a persistent area of research. The results of Gabor's original in-line experiment were marred by the presence of this out of focus twin-image and the so called zero-order intensity terms. While some early attempts at removing these unwanted noise terms were successful ${ }^{2}$ the additional experimental processing involved was laborious and time consuming. Following the invention of the laser an innovative approach to separate the wanted image from the unwanted terms was invented. ${ }^{3}$ This entailed the use of an off-axis reference beam at some angle to the incident object wavefield. The new architecture proved to be a resounding success but notably increased the need for recording materials with higher resolution capacities.

Despite the obvious advantages of the off-axis method there remain a number of holographic applications for which it is either undesirable or is without implementation. The original in-line architecture remains the mode of choice in many cases such as electron holography, x-ray holography and gamma ray holography ${ }^{4}$ due to the lack of physical elements, e.g. an x-ray lens. In the case of optical digital holography ${ }^{4-9}$ the off-axis method has been successfully proposed and investigated. ${ }^{6}$ However, the inherent high resolution requirements of the off-axis technique impose a severe demand on the relatively low resolution of current digital cameras. The resulting restrictions include the placement of macroscopic objects at large distances from the camera and the permission of a very limited range of reconstruction angles (approximately 1-2 degrees for an acceptable image resolution). These parameters can be improved upon by an integer factor if an in-line set-up is used but this introduces the unwanted noise terms.

Removal of the twin-image in digital holography, optical or otherwise, can be broken down into at least five main groups; (i) Off-axis techniques, ${ }^{3,6}$ (ii) Phase shifting interferomtery (PSI), ${ }^{5}$ (iii) Linear filtering ${ }^{7}$ (iv) Phase retrieval methods ${ }^{4}$ and (v) filtering of the complex wavefront in reconstruction planes ${ }^{8,9}$ Phase-shifting requires multiple captures with different phase shifts of the reference beam and is therefore not ideal for single

Further author information: (Send correspondence to C. McElhinney or T. Naughton) E-mail: conormc@cs.nuim.ie, tomn@cs.nuim.ie

Optics and Photonics for Information Processing II, edited by Abdul Ahad Sami Awwal,

Khan M. Iftekharuddin, Bahram Javidi, Proc. of SPIE Vol. 7072, 707208, (2008)

0277-786X/08/\$18 - doi: 10.1117/12.795894

Proc. of SPIE Vol. 7072 707208-1 
capture applications. Linear filtering can work with a single capture but it is generally limited to real objects and is often not as successful as (i) and (ii). Phase retrieval can also work with a single capture but generally requires an intensive iterative computational process, the convergence of which is not always guaranteed. The method outlined in this paper falls into category (v). It requires only a single capture and no additional experimental processing. It can be performed rapidly and shows results comparable with the best of the other methods.

\section{TWIN REDUCTION BY FILTERING THE RECONSTRUCTION PLANE OF DIGITAL HOLOGRAMS}

In [ 9] the first instance of filtering the complex wavefront in the reconstruction planes of digital holograms appeared in the literature. This involved cutting out the wanted digitally reconstructed image from its surrounding pixels. However this area still contained considerable noise from the unwanted twin-image. In [6] spatial filtering was applied to an off-axis digital hologram. In [8] a novel method of filtering the complex wavefront in the reconstruction domain was proposed and it is this method that we build upon in this paper. It was shown that by cutting out the reconstructed focused unwanted twin and returning to the plane of the wanted image by numerical propagation one could free oneself of the unwanted noise. The method was proposed only in the area particle holography and the removal of the twin-images was a manual operation. In this paper we propose the use of a similar technique for macroscopic objects and we make the significant addition of using automatic segmentation algorithms to remove the unwanted macroscopic image. While this paper focuses on optical digital holography, we note that it may be applied to any of the other holographic fields listed above.

Several new approaches to the segmentation of holographic reconstructions into a compact representation of the useful information held in these reconstructions have been developed. ${ }^{10-14}$ These approaches have used phase, ${ }^{12}$ intensity ${ }^{10,14}$ and complex information ${ }^{13}$ as well as the estimated depth ${ }^{11}$ to segment a reconstruction into object(s) and background. They can be further refined into approaches which segment using a single reconstruction ${ }^{10,12}$ or multiple independently focused reconstructions. ${ }^{11,13,14}$ Most of these methods have been developed for segmenting reconstructions containing microscopic objects and plankton. The reconstructions of these near 2D biological organisms are relatively free of speckle noise compared to reconstructions of macroscopic objects encoded in DHs. In this paper we will use a modified version of the object segmentation algorithm ${ }^{14}$ developed for macroscopic objects, of which speckle is a fundamental characteristic.

We use the Fresnel approximation, the propagation transfer function, to numerically propagate our digital holograms. ${ }^{15}$ This is a lossless transform and ensures that the energy of the object signal is preserved after propagation. Due to its use of the discrete Fourier transform with finite support and the conservation of energy the object signal will be wrapped within the reconstruction window. We therefore have to pad at the hologram plane sufficiently that the reconstruction window is larger than the spatial extent of the object signal. This is evident from Fig. 1 where the original hologram data was $2048 \times 2048$ and is centered within the new $8196 \times 8196$ hologram plane. This introduces a computational overhead onto that process which we have addressed through the implementation of the technique on a Graphics Processing Unit (GPU).

\section{THE ALGORITHM}

Our algorithm requires three inputs: a dc-term suppressed hologram $H^{\mathrm{TI}}(x, y)$ in which the dc or zero order term has been removed by some means, the depth, $d \mathrm{~mm}$, of the focal plane of the twin-image, and a block size $n \times n$. Our algorithm can be described in three individual stages, and is displayed in Fig.1

\subsection{Stage 1: Propagate to the twin-image plane}

The first stage requires us to numerically propagate our hologram to the focal plane of the unwanted twin-image using our input hologram $H^{\mathrm{TI}}(x, y)$ and the distance $d \mathrm{~mm}$. This reconstruction is stored in $R^{\mathrm{TI}}(x, y)$ and it's intensity is used to calculate the segmentation mask. 


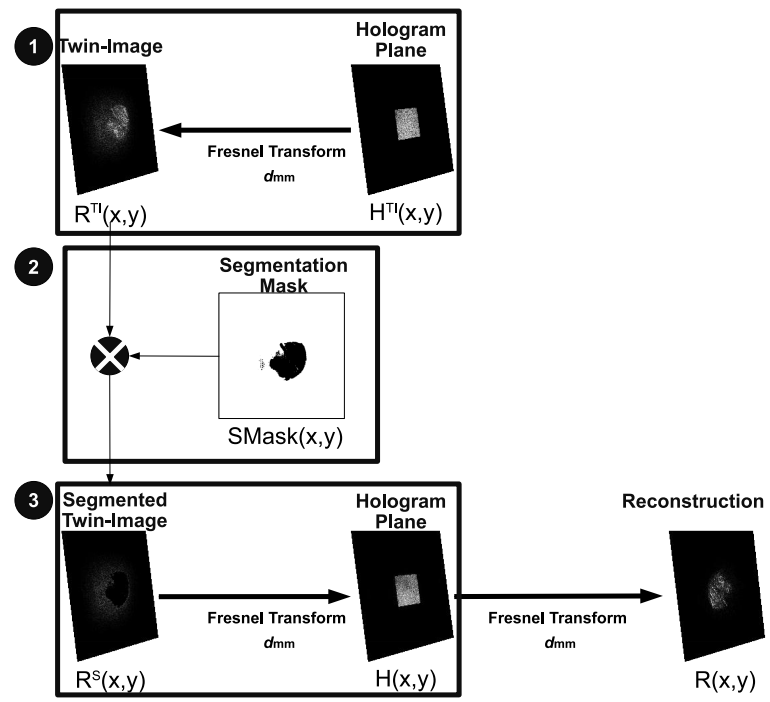

Figure 1. Twin-image removal process stages: 1) propagate to the twin-image plane, 2) segment twin-image and 3) propagate to the hologram plane.

\subsection{Stage 2: Segment twin-image}

To segment the twin-image we need to calculate a segmentation mask $\operatorname{SMask}(x, y)$. We use a modified version of the segmentation approach developed for digital holograms containing macroscopic objects. ${ }^{14}$ In this modified approach the segmentation mask is created through the thresholding of a single variance map. The variance map is calculated by processing an in-focus reconstruction. We select a block size of $n \times n$ and process the intensity of our in-focus reconstruction $\left|R^{\mathrm{TI}}(x, y)\right|^{2}$ by calculating variance on the overlapping blocks using:

$$
V(k, l)=\frac{1}{n^{2}} \sum_{x=k-\left\lfloor\frac{n-1}{2}\right\rfloor}^{k+\left\lceil\frac{n-1}{2}\right\rceil} \sum_{y=l-\left\lfloor\frac{n-1}{2}\right\rfloor}^{l+\left\lceil\frac{n-1}{2}\right\rceil}\left[\left|R^{\mathrm{TI}}(x, y)\right|^{2}-\mu\right]^{2},
$$

where $\mu$ is the arithmetic mean of the current block of $n \times n$ pixels and where any indexes $(x, y)$ that go outside the extent of $R^{\mathrm{TI}}(x, y)$ evaluate to 0 . A location with high variance indicates the nearby presence of an object. A threshold $\tau$ is chosen and $V(k, l)$ is transformed as

$$
\operatorname{SMask}(k, l)=0 \text { if } V(k, l)<\tau ; 1 \text { if } V(k, l) \geq \tau,
$$

where 1 denotes a background pixel and 0 denotes an object pixel. The binary image SMask is our segmentation mask and we obtain the segmented twin-image by simulating an inverse aperture using

$$
R^{\mathrm{S}}(x, y)=R^{\mathrm{TI}}(x, y) \cdot \operatorname{SMask}(x, y)
$$

where - indicates pointwise product.

\subsection{Stage 3: Propagate to the hologram plane}

Once we have successfully segmented the twin-image we then propagate $R^{\mathrm{S}}(x, y)$ to the hologram plane, a distance of $d \mathrm{~mm}$. We now have a hologram, $H(x, y)$, free of the twin-image.

The three stages has been implemented using stream programming ${ }^{16}$ and are executed on programmable graphics hardware. This approach has been shown to render images from digital holograms far more efficiently 
than traditional CPU based methods. ${ }^{17}$ We employ the convolution approach, as described in, ${ }^{17}$ directly for light propagation in Stage 1 and Stage 3. Stage 2, the segmentation stage, may present a problem if implemented directly from Eqn. 1, as the overlapping blocks will cause intensive calculations. However, by rewriting the equation as

$$
V(k, l)=\left[\frac{1}{n^{2}} \sum_{x=k-\left\lfloor\frac{n-1}{2}\right\rfloor}^{k+\left\lceil\frac{n-1}{2}\right\rceil} \sum_{y=l-\left\lfloor\frac{n-1}{2}\right\rfloor}^{l+\left\lceil\frac{n-1}{2}\right\rceil}\left|R^{\mathrm{TI}}(x, y)\right|^{4}\right]-\mu^{2},
$$

we see that it consists of the difference between two sums over the same block area, the mean squared intensities, in brackets, and the squared mean intensities, $\mu^{2}$. We thus divide Stage 2 into two steps. First two summed area tables $^{18}$ are computed, one for each sum in the equation. Second, the variance for a specific location is computed as the difference of the appropriate block areas in the two tables. Our PC has a $2 \mathrm{Ghz}$ core due processor with $2 \mathrm{~GB}$ of RAM and a Nvidia GeForce $8800 \mathrm{GTX}$ with $768 \mathrm{MB}$ of RAM. Using traditional numerical propagation and Eqn. 1 on a CPU the process takes over 2000 seconds for a hologram padded to $4096 \times 4096$. Due to memory requirements this was the largest hologram we could reconstruct with the CPU. However, using the described GPU implementation (and less RAM) we could pad the hologram to the needed $8196 \times 8196$ samples (to guarantee that no wrapping of the object signal) and remove the twin-image in just 8.4 seconds as shown in Table 1.

Table 1. Twin-image removal computation time (seconds)

\begin{tabular}{|l||c|c|}
\hline Process & CPU time & GPU time \\
\hline Pixel Resolution & $4096 \times 4096$ & $8192 \times 8192$ \\
\hline \hline Stage 1 & 25 & 3.4 \\
\hline Stage 2 & 1992 & 1.59 \\
\hline Stage 3 & 25 & 3.4 \\
\hline \hline Total & 2042 & 8.4 \\
\hline
\end{tabular}

\section{RESULTS}

We verify our twin-image removal technique using a DH of a real-world object. The knight object was positioned approximately $377 \mathrm{~mm}$ from the camera with a diameter of $17.5 \mathrm{~mm}$. Our CCD has $2048 \times 2048$ pixels and we have padded the hologram plane to $8192 \times 8192$ pixels. A reconstruction of the hologram before suppression of the dc-term is shown in Fig. 2(a). We manually suppress the dc-term through applying a high pass filter to the Fourier transformed hologram and inverse Fourier transforming the result. ${ }^{6,19}$ We selected a circular aperture with a radius of 200 pixels for this hologram. The reconstruction after dc-term suppression is shown in Fig. 2(b). Our second input was selected by qualitatively determining the depth of the in-focus plane, we selected a depth of $377 \mathrm{~mm}$. Our final input is the block size, $81 \times 81$. We display the calculated segmentation mask in Fig. 2(c) and the output reconstruction after twin-image removal in Fig. 2(d). In the future we intend to compare our twin reduced reconstructions with PSI holograms. PSI holograms are theoretically free of the dc-term and twin-image and a comparison of the PSI reconstruction with the reconstruction from our new approach shows similar quality.

Due to the large hologram size we decided to use the simplified segementation mask creation process outlined in this paper instead of the more exhaustive approach from [14]. For objects with a large depth-of-focus it may be necessary to use the depth-independent segmentation approach from [14]. The accuracy of our approach is currently limited by the manual selection of a threshold in to create the segmentation mask and the manual selection of the twin-image in-focus plane. We have demonstrated the rapid removal of the twin-image from a in-line digital hologram using only digital processing, removing the need for the use of experimental processing, multiple captures or an off-axis experimental setup. 


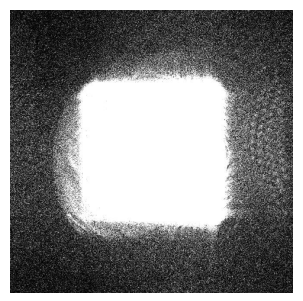

(a)

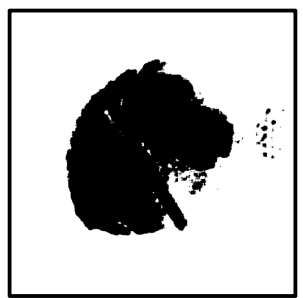

(c)

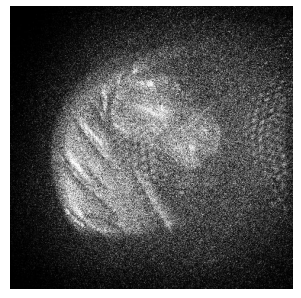

(b)

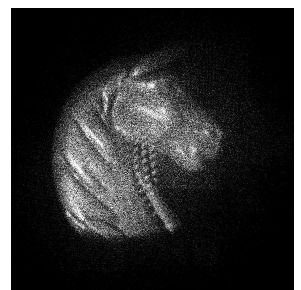

(d)

Figure 2. Numerical reconstructions of knight hologram, reconstruction of (a) unprocessed hologram, (b) hologram after dc-term removal, (c) calculated segmentation mask,(d) hologram after dc-term and twin-image removal

\section{CONCLUSION}

In holography, the in-line architecture offers significant advantages over its off-axis counterpart. In particular we can obtain a higher bandwidth signal and greatly reduce the so-called key-hole problem. However the presence of the twin for the in-line architecture often forces the use of the off-axis set-up. In this paper we have proposed and investigated a novel digital signal processing method for the reduction of twin-image noise from digital Fresnel holograms. We have demonstrated how to firstly reconstruct the unwanted twin-image when it is in-focus and in this plane we calculate a segmentation mask that borders this in focus image. Care must be taken to ensure that we can digitally calculate the full support of the this plane. This is accomplished by considerable zero padding in the reconstruction algorithm. The resultant increased computation is dealt with by employing dedicated hardware devices. The twin-image is then segmented and removed by simple spatial filtering. The resulting digital wavefield is the inverse propagated to the desired object image plane. The image is free of the twin-image resulting in improved quality reconstructions. We have demonstrated the validity of the method and outlined times taken for its implementation.

\section{ACKNOWLEDGMENTS}

The research leading to these results has received funding from the European Community's Seventh Framework Programme FP7/2007-2013 under grant agreement no. 216105.

\section{REFERENCES}

1. D. Gabor, "A new microscopic principle," Nature 161, p. 777, 1948.

2. W. Bragg and G. Roger, "Elimination of the unwanted image in diffraction microscopy," Nature 167, p. 190, 1951.

3. E. Leith and J. Upatnieks, "Wavefront reconstruction with continuous-tone objects," J. Opt. Soc. Am. 53, pp. 1377-1381, 1963.

4. T. Latychevskaia and H. Fink, "Solution to the twin image problem in holography," Phys. Rev. Lett. 98, p. 23390, 2007.

5. I. Yamaguchi and T. Zhang, "Phase-shifting digital holography," Opt. Lett. 22, p. 1268, 1997.

6. E. Cuche, P. Marquet, and C. Depeursinge, "Spatial filtering for zero-order and twin-image elimination in digital off-axis holography," Appl. Opt. 39, pp. 4070-4075, 2000. 
7. L. Onural and P. Scott, "Digital decoding of in-line holograms," Opt. Eng. 26, pp. 1124-1132, 1987.

8. L. Denis, C. Fournier, T. Fournel, and C. Ducottet, "Twin-image noise reduction by phase retrieval in in-line digital holography," Proc. SPIE 5914, pp. 59140J-1, 2007.

9. J. Pedrini, P. Frning, H. Fessler, and H. Tiziani, "In-line digital holographic interferometry," Appl. Opt. 37, pp. 6262-6269, 1998.

10. P. Hobson and J. Watson, "The principles and practice of holographic recording of plankton," J. Opt. A: Pure Appl. Opt. 4, p. S32, 2002.

11. E. Malkiel, J. Abras, and J. Katz, "Automated scanning and measurement of particle distributions within a holographic reconstructed volume," Meas. Sci. and Tech. 15, pp. 601-612, 2004.

12. M. Gustafsson and M. Sebesta, "Refractometry of microscopic objects with digital holography," Appl. Opt. 43, pp. 4796-4801, 2004.

13. M. DaneshPanah and B. Javidi, "Segmentation of 3d holographic images using bivariate jointly distributed region snake," Opt. Exp. 14, pp. 5143-5153, 2006.

14. C. McElhinney, J. McDonald, A. Castro, Y. Frauel, B. Javidi, and T. Naughton, "Depth-independent segmentation of three-dimensional objects encoded in single perspectives of digital holograms," Opt. Lett. 32, pp. 1129-1231, 2007.

15. T. Kreis, Handbook of Holographic Interferometry, Wiley-Vch, New York, 2005.

16. U. Kapasi, S. Rixner, W. Dally, B. Khailany, J. Ahn, P. Mattson, and J. Owens, "Programmable stream processors," IEEE Computer.

17. L. Ahrenberg, A. Page, B. Hennelly, J. McDonald, and T. Naughton, "Using commodity graphics hardware for real-time digital hologram view reconstruction," in preparation .

18. F. Crow, "Summed-area tables for texture mapping," SIGGRAPH Comput. Graph. 18, pp. 207-212, 1984.

19. G. Chen, C. Lin, M. Kuo, and C. Chang, "Numerical suppression of zero-order image in digital holography," SIGGRAPH Comput. Graph. 15, pp. 8851-8856, 2007. 